\title{
Note on Transliteration and Translation
}

When transliterating from Russian to English, I have used the Library of Congress system with occasional modifications when dealing with names of persons and places that will be familiar to Anglophone readers. Therefore, for example, I write Tolstoy instead of Tolstoi, Petersburg instead of Peterburg, and Ilya instead of Il'ia. Throughout the notes and bibliography, I have adhered strictly to the Library of Congress transliteration standards.

Unless indicated otherwise, all translations are mine. 
